\title{
Visibilidade de revistas científicas: um estudo no Portal de Periódicos Científicos da Universidade Federal do Rio Grande do Sul
}

\author{
Visibility of scientific journals: A study based on the \\ Website of Scientific Journals at Universidade \\ Federal do Rio Grande do Sul
}

\author{
Ana Gabriela Clipes FERREIRA' \\ Sônia Elisa CAREGNATO²
}

\section{Resumo}

Esta pesquisa analisa a visibilidade e as características que promovem as revistas científicas eletrônicas, presentes no Portal de Periódicos Científicos da Universidade Federal do Rio Grande do Sul. Avalia-se, também, o grau de presença dessas revistas nas fontes de informação da internet. Compreende-se, portanto, que este é um estudo descritivo e exploratório, que combina dados qualitativos e quantitativos para analisar o corpus formado por 34 títulos. O mapeamento das fontes de informação permitiu-nos destacar a maior presença de periódicos na Latin American Periodicals Tables of Contents, seguida pela Latindex. Apesar da presença pouco expressiva em fontes de informação internacionais, concluiu-se que as revistas pertencentes ao Portal de Periódicos Científicos buscam adaptar-se às características que promovem sua visibilidade. O trabalho também sugere uma série de novos estudos, que visariam aprofundar as análises de outros temas ligados à visibilidade e aos estudos bibliométricos.

Palavras-chave: Comunicação científica. Fontes de informação. Periódicos. Visibilidade.

\begin{abstract}
The study addresses the visibility of electronic scientific journals, as well as the characteristics that promote, especially focusing on those hosted on the Portal of Scientific Journals of the Universidade Federal do Rio Grande do Sul. The study assesses the degree of visibility of these journals on the Internet by means of their presence in information sources. The theoretical reference addresses the following themes: scientific communication; scientific journals; visibility and searchability; information sources on portal; bibliographic databases and open access. This is a descriptive and exploratory study that combines qualitative and quantitative data. The corpus of research was composed of 34 titles. Essential information about the journals was collected, giving emphasis to the characteristics that promote their visibility. It was found that Human Sciences is the area with the greatest number of journals at the University, 29.4\% of all publications. Open access is adopted by all journals in the sample. Once the information sources had been mapped, it was found that the majority of journals appear in the information source Latin American Periodicals Tables of Contents, followed by Latindex. Despite the limited presence of the journals in international information sources, it was concluded that the journals hosted on the Website of Scientific Journals seek to adapt to characteristics that promote their visibility. The study suggests a number of new studies, deepens the analyses, and addresses other themes related to visibility and, especially, bibliometric studies.
\end{abstract}

Keywords: Scientific communication. Information sources. Journal. Visibility.

\footnotetext{
1 Universidade Federal do Rio Grande do Sul, Faculdade de Educação, Biblioteca Setorial de Educação. Av. Paulo Gama, 110, Prédio 12201, Farroupilha, 90046-900, Porto Alegre, RS, Brasil. Correspondência para/Correspondence to: A.G.C. FERREIRA.E-mail:<anaclipes@ufrgs.br>.

2 Universidade Federal do Rio Grande do Sul, Faculdade de Biblioteconomia e Comunicação, Departamento de Ciência da Informação. Porto Alegre, RS, Brasil.

Recebido em 30/10/2012 e aceito para publicação em 20/2/2014.
} 


\section{Introdução}

As Tecnologias da Comunicação e da Informação (TIC) trouxeram novidades aos processos de comunicação e divulgação da ciência, uma vez que a presença na web ampliou o acesso aos resultados de pesquisa documentados e promoveu o uso dessa produção. As revistas eletrônicas estão disponíveis na Web pelas diferentes fontes de informações, quais sejam: os portais, os índices, as bases de dados, os repositórios e os diretórios. Essas fontes de informação na Web apresentam recursos que facilitam a localização de artigos dos periódicos nelas indexados e, portanto, promovem a visibilidade desses materiais. Um periódico se torna visível na ciência quando possui uma série de características que promovam o seu acesso, ou seja, quando as informações têm a capacidade de exposição, influenciando, assim, o público-alvo (Packer \& Meneghini, 2006).

Visto que existe um número elevado de títulos de periódicos eletrônicos, se comparados, os periódicos que possuírem determinados atributos serão mais visíveis. Entre as características que proporcionam visibilidade é possível destacar: a presença na Web, o acesso aberto, a buscabilidade, o idioma da publicação e a presença em fontes de informação. Assim, formula-se a seguinte questão: quais são as características promotoras de visibilidade que os periódicos científicos eletrônicos podem possuir?

Nesse sentido, este artigo indaga se somente a presença em bases de dados bibliográficas internacionais é capaz de conferir visibilidade aos periódicos, bem como quais seriam as características que tornam um periódico visível. Parte-se da premissa de que a visibilidade não é formada por um único atributo.

O objetivo geral, portanto, é analisar a relação entre as características dos periódicos científicos eletrônicos presentes no Portal de Periódicos Científicos da Universidade Federal do Rio Grande do Sul (PPCU/UFRGS) e a visibilidade que alcançam. Paralelamente, a fim de subsidiar o alcance desse objetivo, salientam-se as principais características dos periódicos: o idioma, o acesso aberto e a avaliação no Qualis da Coordenação de Aperfeiçoamento de Pessoal de Nível Superior (CAPES). Além disso, foi realizado o mapeamento das fontes de informação eletrônicas nas quais os periódicos estão presentes. Por fim, apresentam-se considerações acerca da visibilidade dos periódicos científicos eletrônicos.

\section{Visibilidade e os atributos que a promovem}

O termo visibilidade possui na literatura especializada diferentes definições. Para Packer e Meneghini (2006, p.237) visibilidade é a"[...] capacidade de exposição que uma fonte ou fluxo de informação possui de, por um lado, influenciar seu público-alvo e, por outro, ser acessada em resposta a uma demanda de informação". Para ser visível, o periódico eletrônico precisa atingir membros de uma comunidade, que buscam sanar suas demandas e necessidades informacionais. Nesse caso, a visibilidade de uma publicação científica indica o seu reconhecimento.

Para Zimba e Mueller (2004, p.49), visibilidade científica "é o grau de exposição e evidência de um pesquisador frente à comunidade científica", sendo que trabalhos e ideias de um pesquisador que sejam facilmente acessíveis podem ser considerados detentores de uma alta posição de visibilidade. A definição é voltada para a visibilidade do autor e não leva em consideração o reconhecimento ou a qualidade do seu trabalho, já que a exposição pode ser negativa.

Para os periódicos alcançarem visibilidade precisam ser referência de qualidade e credibilidade em uma determinada área e estar indexados em índices de prestígio nacionais e internacionais (Packer \& Meneghini, 2006). Partindo dessas duas dimensões, Packer e Meneghini (2006) propõem alguns atributos e indicadores de visibilidade, que são: (a) sociedade científica, organização, grupo ou empresa editora responsável pela publicação do periódico; (b) representatividade do editor responsável e do corpo editorial junto à comunidade científica; (c) distribuição institucional e geográfica da afiliação dos autores; (d) publicação eletrônica na Internet e com acesso aberto; (e) idioma(s) de publicação; (f) número de índices referenciais nacionais e internacionais que está indexado; (g) números médios de leituras ou de artigos acessados periodicamente; (h) número de citações recebidas e fator de impacto.

Villamón et al. (2009) afirmam que, para haver disseminação da informação dos periódicos, é necessária a visibilidade na Internet e a indexação em bases de dados, preferencialmente internacionais. Porém, o 
conceito de visibilidade para esses autores se limita a esses dois indicadores. Em estudo que caracterizou as revistas espanholas presentes na Latindex, a análise de visibilidade dos autores deu-se pela análise do conteúdo disponibilizado na Internet, ou seja, se determinada revista disponibiliza ou não os artigos em formato eletrônico (Villamón et al., 2009).

Certamente, a presença na Web e em índices e bases de dados, ou de uma maneira mais ampla, a presença em fontes de informação eletrônicas, são itens essenciais para a promoção da visibilidade. Todavia, o conjunto de atributos propostos por Packer e Meneghini (2006) englobam essas características e acrescentam outras importantes.

Pode-se inferir que as condições básicas para se obter visibilidade são a aceitação, o reconhecimento, o uso, a credibilidade e o acesso à informação. É importante salientar que nem todas essas condições precisam estar presentes ao mesmo tempo para definir se um periódico é visível. Por esse motivo, por possuírem uma estreita ligação e dependência, em determinadas ocasiões, pode ser entendido que os itens ou os atributos que tornam a informação visível sejam definidos como visibilidade.

Assim, neste trabalho aborda-se a visibilidade dos periódicos científicos eletrônicos com ênfase em determinados atributos. As características foram baseadas no trabalho de Packer Meneghini (2006), quais sejam: publicação em revistas de acesso aberto na Internet, idioma de publicação da revista, e os índices e bases de dados nacionais e internacionais que as indexam. Esses itens foram selecionados por serem os mais complexos para a avaliação dos periódicos científicos. Os demais atributos - a sociedade científica, a organização, o grupo ou empresa editora responsável pela publicação do periódico e pela representatividade do editor ou do corpo editorial, bem como a distribuição institucional e geográfica de afiliação dos autores -, não serão considerados por serem características empregadas no instante em que a revista é incluída nos índices e na base de dados.

O termo searchability, ou "buscabilidade" quando traduzido para o português, se refere à capacidade do site de ser encontrado pelos principais mecanismos de busca; conjuntamente, representa a possibilidade de o usuário acessar e identificar a informação recuperada (Hernández Ortega et al., 2010), sendo, nesse sentido, similar aos conceitos de visibilidade.

Estudos sobre a buscabilidade são recentes e alguns foram relacionados ao uso de redes sociais; por esse motivo, o termo é considerado um neologismo da Internet. Principalmente, observa-se a utilização da buscabilidade em estudos da área de Sistemas de Informação, para avaliar desenhos de sites e a influência do tráfego de informações. Porém, já é possível observar alguns estudos nas Ciências Sociais Aplicadas também.

Peazê (2009, online) define buscabilidade como a "característica ativa ou passiva de buscar ou encontrar informação ou dado na Internet", ou seja, para ele trata-se de uma definição ligada diretamente aos mecanismos de busca. Assim, verifica-se que visibilidade e buscabilidade não são termos sinônimos, embora tenham aspectos comuns. No contexto da comunicação científica, a visibilidade é um conjunto de características e atributos de um pesquisador ou periódico, enquanto a buscabilidade é a capacidade de recuperação da informação em mecanismos de busca. Porém, a possibilidade de recuperar um título utilizando esses recursos é mais um dos itens que proporcionam a visibilidade da revista. Assim, buscabilidade é uma característica adicional de visibilidade.

\section{Fontes de informação e presença na Web}

As publicações periódicas, ao migrarem para o meio eletrônico, promoveram mudanças não só de formato, influenciaram, também, a criação de novos serviços para disponibilizá-las, e promoveram a adaptação de outros já existentes, são eles: os índices e os serviços de indexação e resumo, ou, como também são denominadas, as bases de dados bibliográficos.

Bases de dados e índices são produtos de serviços de indexação e resumo, responsáveis pela sua divulgação e comercialização (Céndon, 2000). A indexação é uma etapa na produção desses serviços de índice e resumo oferecidos ao público na forma de base de dados de acesso online, e não mais na forma impressa. Silva et al. 
(2006, p.264) afirmam que bases de dados eletrônicas são as fontes de informação "pesquisáveis de modo interativo ou conversacional através de um computador".

As bases de dados podem ser classificadas de acordo com a natureza da informação nelas presentes. Rowley (2002) faz a divisão e subdivisão dos tipos de bases de dados da seguinte maneira: (a) bases de dados de referências ou referenciais (bibliográficas, catalográficas e de diretórios); (b) bases de dados de fontes (numéricas, de texto completo, textuais e numéricas e de gráficos).

Packer e Meneghini (2006) afirmam que um dos atributos de visibilidade é a quantidade de fontes de informação na qual os periódicos estão presentes. Porém, nem todas as fontes possuem a mesma importância para a área do conhecimento, pois a cobertura e abrangência são diferentes, assim como seus objetivos.

Para Meneghini (1998) a ciência brasileira é comparada a um iceberg: apenas a parte acima da água representa a produção científica indexada em bases de dados internacionais e está visível: o restante da produção não presente é pouco visível à ciência. Em contraponto à realidade das bases de dados internacionais, nas quais a presença de revistas brasileiras, da América Latina e Caribe ainda é escassa, Aguado-López et al. (2010) apontam fontes de informação que têm como objetivo a divulgação do conhecimento científico dessas regiões, considerando-as como indicadores alternativos para a avaliação das atividades científicas em contraponto com o ISI, recurso no qual as revistas norte-americanas e inglesas da área das ciências duras estão mais presentes. Citam projetos como a Scientific Electronic Library Online (SciELO), Red de Revistas Científicas da América Latina y el Caribe, España y Portugal (Redalyc) e Univerciência.org, que são diferentes entre si, pois cobrem áreas do conhecimento e têm objetivos distintos. A Redalyc, por exemplo, indexa 76\% das revistas da área de Ciências Humanas, enquanto na SciELO pode ser observada a predominância da área de Ciências da Saúde, com 40\%. Porém, ambas as fontes permitem a produção de indicadores bibliométricos.

Miguel e Herrero-Solana (2010) também indicam as iniciativas latino-americanas que têm como objetivo aumentar a visibilidade, no requisito presença em índices bibliográficos, dos periódicos da região. Os autores discutem a pouca presença das revistas latino-americanas de Biblioteconomia e Ciência da informação nos grandes serviços de indexação e resumo, além das dificuldades de identificação, valorização da qualidade e determinação da natureza. Essas características, segundo os autores, diminuem as possibilidades de reconhecimento e visibilidade internacional. Além da RedaLyC, a Latindex é um dos projetos apontados como importantes para esse autores, podendo ser uma medida alternativa de presença em fontes de informação. Essas fontes contribuem para a divulgação e disseminação das revistas de diversas áreas do conhecimento. O estudo de Miguel e Herrero-Solana (2010) é mais um em que a visibilidade é diretamente ligada à presença em bases de dados, tendo sido o diferencial à utilização da Latindex como fonte para o estudo. Nesse caso, a escolha da fonte foi importante por se tratar de uma área, as Ciências Sociais Aplicadas, que não possui uma presença marcante nas grandes bases de dados bibliográficas multidisciplinares. O estudo possibilitou uma análise que teria resultado não condizente com a realidade da área.

\section{Métodos}

Trata-se de um estudo descritivo e exploratório, com análise de dados qualitativos e quantitativos. 0 objeto empírico da pesquisa é o grupo de periódicos científicos eletrônicos editados na UFRGS e disponibilizados no Portal de Periódicos Científicos da Universidade. A formação do corpus da pesquisa foi realizada pela análise das revistas pertencentes ao PPCU.

Dos 37 títulos das diversas áreas do conhecimento, foram excluídas apenas três, por se tratarem de revistas de associações. Tais revistas contavam com editor da Universidade na época da coleta de pesquisa, mas o cargo pode ser transferido a qualquer momento para outro docente de diferente instituição, fazendo com que a revista seja retirada do portal. Assim, preferiu-se excluir deste estudo esse tipo de publicação. A consulta aos títulos ocorreu em outubro de 2010.

Para a coleta de dados, foi criado um instrumento em formato de formulário. As informações foram coletadas por intermédio das informações fornecidas no PPCU pelas equipes editoriais das revistas. Quando incompletas, desatualizadas ou mesmo para a conferência 
das informações, utilizou-se os seguintes recursos: (a) consulta à página do periódico; (b) consulta ao Catálogo Online do Sistema de Bibliotecas (SABI) da UFRGS; e (c) consulta ao Catálogo Coletivo Nacional do Instituto Brasileiro de Informação em Ciência e Tecnologia (CCN/IBICT).

A organização dos dados foi realizada em planilha eletrônica. Os recursos oferecidos pelo formato "xls" possibilitaram a melhor categorização das informações e a utilização de recursos como, por exemplo, a organização das listas em ordem alfabética.

Os dados sobre as fontes de informações eletrônicas - indexadores no PPCU -, necessitaram de um número maior de colunas e de limpeza e uniformização de dados. A limpeza desses dados foi realizada, primeiramente, pela padronização dos títulos das bases de dados, dos portais e dos diretórios. Após essa etapa, conferiu-se a presença das revistas nas fontes de informação. Foram contabilizadas as fontes de informação em que está presente o maior número de revistas do PPCU pela contagem de palavras da planilha eletrônica.

As fontes de informação foram listadas de acordo com o número de vezes em que foram citadas, ou seja, se a fonte foi citada por cinco títulos diferentes, por exemplo, na listagem o seu nome apareceu o mesmo número de vezes. O recurso Wordle <http://www.wordle.net/> auxiliou na elaboração de uma nuvem a partir dessa listagem, com todas as ocorrências das fontes nas revistas.

Por fim, a análise sobre visibilidade foi realizada e os resultados foram agrupados de acordo com a tabela do Conselho Nacional de Desenvolvimento Cientifico e Tecnológico (CNPq) das grandes áreas do conhecimento, com o propósito de realizar uma análise geral do conjunto, e não título por título.

\section{As revistas do PPCU}

Os periódicos abordados neste estudo, seus respectivos títulos, área do conhecimento (informada pelos editores das revistas), International Standard Serial Number (ISSN), Eletronic International Standard Serial Number (E-ISSN), periodicidade e ano inicial estão listados no Quadro 1.
A utilização dos padrões de acesso aberto pelas revistas é marcante. Dos 34 títulos, 32 empregam o Sistema de Editoração Eletrônico de Revista/Open Journal Sistems (SEER/OJS) de alguma maneira: três dessas 32 revistas em processo de migração e outras duas utilizam a versão anterior, impossibilitando a presença no Portal SEER/UFRGS. A utilização de página Hypertext Markup Language (HTML) foi observada em quatro títulos, sendo que apenas dois deles não possuem também acesso pelo SEER/OJS. Outros três títulos estão presentes também na SciELO, biblioteca eletrônica que utiliza o software OJS desde 2005 (Santos, 2005). Além disso, observou-se a presença significativa de revistas híbridas. Todavia, vários títulos deixaram de fornecer o formato impresso nos últimos anos e adotaram somente o formato eletrônico.

A presença no Portal de Periódicos da Capes é expressiva. Das revistas analisadas, 23 títulos estão presentes no Portal. Dos 11 títulos que estão fora do portal, alguns deles, como a Educação \& Realidade, têm plenas condições de estar presente no PPC: possui Qualis A2 na área de Educação e está indexado em fontes de informação adequadas e relevantes para a área. O PPC é uma fonte de informação importante por disponibilizar entre os seus recursos periódicos e bases de dados, tanto que é citado por grande parte das revistas do PPCU. Porém, não é uma fonte que indexa os artigos ou fornece outros serviços, como indicadores de produção ou mesmo referências bibliográficas.

Foram encontrados problemas quanto à atualização dos dados no PPCU. Algumas informações estão desatualizadas, outras inexistentes. Quanto à padronização, uma limitação observada foi a ausência do E-ISSN em alguns dos títulos. Sendo revistas eletrônicas, não possuir o número padrão para publicações é uma falha grave e que pode interferir na avaliação da revista, seja por agências de fomento ou pelas fontes de informação, como pelo próprio leitor ou autor em potencial da publicação.

Somente as revistas que estão presentes na SciELO possuem Digital Object Identifier (DOI). O número identificador é um qualificador da publicação ao identificar e localizar objetos na Internet. O fato de a revista possuir esse recurso representa segurança na localização dos documentos eletrônicos, assim como permite a participação em uma rede internacional de dados bibliográficos. 
Quadro 1. Revistas do Portal da Universidade Federal do Rio Grande do Sul. 2010.

\begin{tabular}{|c|c|c|c|c|c|}
\hline Título da Revista & Área do Conhecimento & ISSN & E-ISSN & Periodicidade & Ano inicial \\
\hline Acta Scientiae Veterinariae & Veterinária & $1678-0345$ & $1679-9216$ & Trimestral & 1973 \\
\hline Análise Econômica & Ciências Econômicas & 0102-9924 & $2176-5456$ & Semestral & 1983 \\
\hline Anos 90 & História & $0104-236 x$ & $1983-201 x$ & Semestral & 1993 \\
\hline Arqtexto & Arquitetura & $1518-238 x$ & - & Semestral & 2000 \\
\hline Caderno de Farmácia & Farmácia & $0102-6592$ & - & Semestral & 1985 \\
\hline Cadernos do Aplicação & Educação & 0103-6045 & - & Semestral & 1986 \\
\hline Cena & Teatro & $1519-275 x$ & - & Semestral & 2000 \\
\hline ConTexto & Não informado & $1676-6016$ & $2175-8751$ & Semestral & 2001 \\
\hline Contingentia & Letras & & $1980-7589$ & Semestral & 2006 \\
\hline Debates do NER & Antropologia & $1519-843 x$ & $1982-8136$ & Semestral & 1997 \\
\hline Educação \& Realidade & Educação & $0100-3143$ & $2175-6236$ & Quadrimestral & 1976 \\
\hline Em Pauta & Música & 0103-7420 & $1984-7491$ & Semestral & 1989 \\
\hline Em Questão* & Biblioteconomia e Comunicação & $1807-8893$ & $1808-5245$ & Semestral & 1986 \\
\hline Episteme & Filosofia e Ciências Humanas & $1413-5736$ & - & Semestral & 1996 \\
\hline Espaço Ameríndio & Antropologia & - & $1982-6524$ & Semestral & 2007 \\
\hline Estudos Interdisciplinares sobre o Envelhecimento & Interdisciplinar & $1517-2473$ & - & Semestral & 1999 \\
\hline Horizontes Antropológicos & Antropologia & 0104-7183 & $1806-9983$ & Semestral & 1995 \\
\hline Informática na Educação: Teoria \& Prática & Interdisciplinar & $1516-084 X$ & $1982-1654$ & Semestral & 1998 \\
\hline Intexto & Biblioteconomia e Comunicação & - & $1807-8583$ & Semestral & 1997 \\
\hline Movimento & Educação Física & $0104-754 x$ & $1982-8918$ & Trimestral & 1994 \\
\hline Nau Literária & Letras & & $1981-4526$ & Semestral & 2005 \\
\hline Para Onde!? & Geociências & & $1982-0003$ & Semestral & 2007 \\
\hline Pesquisas em Geociências ${ }^{* *}$ & Geociências & $1518-2398$ & $1807-9806$ & Semestral & 1972 \\
\hline Porto Arte & Artes visuais & $0103-7269$ & & Semestral & 1990 \\
\hline Produto e Produção & Interdisciplinar & 15163660 & $1983-8026$ & Quadrimestral & 1997 \\
\hline Psicologia: reflexão e crítica & Psicologia & $0102-7972$ & $1678-7153$ & Quadrimestral & 1986 \\
\hline REAd: Revista Eletrônica da Administração & Administração & $1980-4164$ & $1413-2311$ & Quadrimestral & 1995 \\
\hline Revista Brasileira de Biociências & Biociências & $1679-2343$ & $1980-4849$ & Trimestral & 2003 \\
\hline Revista da Faculdade de Odontologia & Odontologia & $0566-1854$ & - & Quadrimestral & 1960 \\
\hline Revista de Informática Teórica e Aplicada & Informática & 0103-4308 & - & Semestral & 1989 \\
\hline Revista Debates & Filosofia e Ciências Humanas & & $1982-5269$ & Semestral & 2007 \\
\hline Revista Gaúcha de Enfermagem & Enfermagem & $0102-6933$ & $1983-1447$ & Trimestral & 1976 \\
\hline Revista HCPA & Medicina & 0101-5575 & $1983-5485$ & Quadrimestral & 1981 \\
\hline Sociologias & Sociologia & $1517-4522$ & $1807-0337$ & Semestral & 1999 \\
\hline
\end{tabular}

Notas: " continuação da Revista de Biblioteconomia, a partir de 2003; ${ }^{* *}$ continuação da Revista Pesquisas, a partir de 2000.

ISSN: International Standard Serial Number; E-ISSN: Eletronic International Standard Serial Number.

Fonte: Elaborado pelas autoras (2010).

Quanto ao idioma, a maioria dos periódicos publica artigos em português. Observa-se na literatura a importância da adoção do idioma inglês para que se tenha ampla aceitação pelas comunidades científicas. Há a presença de artigos em outros idiomas, em especial o espanhol. Também há artigos em inglês, francês e mesmo em alemão na revista Contigentia. Contudo, observou-se que a única revista que publica exclusivamente em inglês é a Acta Scientiae Veterinariae. As demais disponibilizam resumo e palavras-chave na língua inglesa. Essa 
Quadro 2. Fontes de informações das Revistas do Portal de Periódicos Científicos da Universidade Federal do Rio Grande do Sul. 2010.

\begin{tabular}{l}
\hline Fonte \\
\hline Bases de dados re \\
\hline Base de Dados em Enfermagem (BDENF) \\
Bibliografia Brasileira de Educação (BBE) \\
Bibliografia Brasileira de Odontologia (BBO) \\
Bibliography and Index of Geology \\
Base de Dados Referenciais de Artigos de Periódicos em Ciência da \\
Informação (BRAPCI) / Base \\
CABI International / CABI International - Index Veterinarius \\
Cambridge Scientific Abstracts (CSA), Social Services Abstracts e \\
Sociological Abstracts \\
Cumulative Index to Nursing \& Allied Health Literature (CINAHL)
\end{tabular}

Citas Latinoamericanas en Sociologia, Economiay Humanidades (CLASE)

Cuiden

Data Índice - Instituto Universitário de Pesquisas do Rio de Janeiro (IUPERJ)

Digital Bibliography \& Library Project (DBLP)

Georef

Index Medicus Latino-Americano

Index-Psi Periódicos

Índice de Aquitetura da USP

International Nursing Índex

Indice de Revistas en Educación Superior y Investigación Educativa (IRESIE)

ISI Web of Knowledge

Latin American Periodicals Tables of Contents (LAPTOC)

Centro Latino-Americano e do Caribe de Informação em Ciências da Saúde (LILACS)

MedLine

Mineralogical Abstracts

Orientador Adviser - Infobase IBBA - Índice Brasileiro de Bibliografia de Administração

The Philosopher's Índex

Psicodoc - Colegio Oficial de Psicólogos de Madrid

Psyclnfo (Psychological Abstracts)

1 de 3

Acta Scientiae Veterinariae, Revista Brasileira de Biociências

Episteme, Psicologia: Reflexão e Crítica, Sociologias

Revista Gaúcha de Enfermagem

Educação \& Realidade, Estudos Interdisciplinares sobre o Envelhecimento, Psicologia: Reflexão e Crítica, Sociologias

Revista Gaúcha de Enfermagem

Sociologias

Revista de Informática Teórica e Aplicada - RITA

Pesquisas em Geociências

Revista Gaúcha de Enfermagem

Estudos Interdisciplinares sobre o Envelhecimento, Psicologia: Reflexão e Crítica

Arqtexto

Revista Gaúcha de Enfermagem

Educação \& Realidade

Acta Scientiae Veterinariae, Movimento, Psicologia: Reflexão e Crítica Acta Scientiae Veterinariae, Análise Econômica, Anos 90, Arqtexto, Caderno de Farmácia, Cadernos do Aplicação, ConTexto, Contingentia, Debates do NER, Educação \& Realidade, Em Pauta, Em Questão, Estudos Interdisciplinares sobre o Envelhecimento, Horizontes Antropológicos, Informática na Educação: Teoria \& Prática, Movimento, Pesquisas em Geociências, Porto Arte, Produto \& Produção, Psicologia: Reflexão e Crítica, Revista Brasileira de Biociências, Revista da Faculdade de Odontologia, Revista Gaúcha de Enfermagem

Estudos Interdisciplinares sobre o Envelhecimento, Movimento, Psicologia: Reflexão e Crítica, Revista da Faculdade de Odontologia, Revista Gaúcha de Enfermagem, Revista HCPA

Revista Gaúcha de Enfermagem

Pesquisas em Geociências

REAd: Revista Eletrônica da Administração

Episteme

Psicologia: Reflexão e Crítica

Psicologia: Reflexão e Crítica 
Quadro 2. Fontes de informações das Revistas do Portal de Periódicos Científicos da Universidade Federal do Rio Grande do Sul. 2010.

\begin{tabular}{|c|c|}
\hline Fonte & Revista \\
\hline \multicolumn{2}{|c|}{ Bases de dados referenciais bibliográficas } \\
\hline $\begin{array}{l}\text { Red de Revistas Científicas da América Latina yel Caribe, Españay Portugal } \\
\text { (RedALyC) }\end{array}$ & Movimento, Psicologia: Reflexão e Crítica \\
\hline Portal de Revistas de Enfermagem (Rev@Enf) & Revista Gaúcha de Enfermagem \\
\hline Scopus & Psicologia: Reflexão e Crítica, Revista Gaúcha de Enfermagem \\
\hline $\begin{array}{l}\text { Sistema Brasileiro de Documentação e Informação Desportiva } \\
\text { (SIBRADID) }\end{array}$ & Estudos Interdisciplinares sobre o Envelhecimento \\
\hline SOCINDEX & Sociologias \\
\hline SPORTDiscus & Movimento \\
\hline Child Development Abstracts and Bibliography (SRCD) & Psicologia: Reflexão e Crítica \\
\hline Sumários de Revistas Brasileiras & $\begin{array}{l}\text { Contingentia, Espaço Ameríndio, Estudos Interdisciplinares sobre o } \\
\text { Envelhecimento, REAd: Revista Eletrônica da Administração, Revista } \\
\text { Debates }\end{array}$ \\
\hline \multicolumn{2}{|c|}{ Bases de dados referenciais catalográficas } \\
\hline Bauhaus-Universität Weimar & Contingentia \\
\hline Biblioteca Revistas Eletrônicas CCG/IBT & Contingentia \\
\hline Biblioteques UAB. Revistes Digitals & Psicologia: Reflexão e Crítica \\
\hline Elektronische Zeitschriftenbibliothek (EZB) & Contingentia \\
\hline Fundação Biblioteca Nacional (FBN) & Arqtexto \\
\hline Fundação Carlos Chagas (FCC) & Educação \& Realidade \\
\hline Germanistik im Netz & Contingentia \\
\hline Library of Congress (LOC) & Arqtexto \\
\hline Library University Georgetown & Contingentia \\
\hline The Royal Institute of British Architects (RIBA) & Arqtexto \\
\hline York University Libraries & Contingentia \\
\hline
\end{tabular}

Bases de dados fontes

\begin{tabular}{cc}
\hline Scientific Electronic Library Online (SciELO) & Horizontes Antropológicos, Psicologia: Reflexão e Crítica, Sociologias \\
\hline Diretórios de revistas, portais e outras fontes de informação
\end{tabular}

Directory of Open Access Journals (DOAJ)

Contingentia, Espaço Ameríndio, Pesquisas em Geociências, Psicologia: Reflexão e Crítica, Revista Brasileira de Biociências, Revista Debates

184

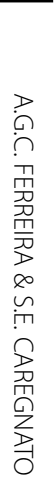

\section{E-Journals.org}

Google Acadêmico

Sistema Regional de Información en Línea para Revistas Científicas de América Latina, el Caribe, España y Portugal (Latindex)
Revista Brasileira de Biociências

Pesquisas em Geociências, Revista Brasileira de Biociências, Revista de Informática Teórica e Aplicada - RITA

Anos 90, Arqtexto, Caderno de Farmácia, Contingentia, Educação \& Realidade, Em Pauta, Em Questão, Espaço Ameríndio, Estudos Interdisciplinares sobre o Envelhecimento, Movimento, Psicologia: Reflexão e Crítica, REAd: Revista Eletrônica da Administração, Revista Brasileira de Biociências, Revista Debates, Revista Gaúcha de Enfermagem, Revista HCPA, Sociologias

Em questão 


\begin{tabular}{cc}
\hline Fonte & Revista \\
\hline & Diretórios de revistas, portais e outras fontes de informação \\
\hline
\end{tabular}

Open Archives Harvester - Public Knowledge Project (OAH/PKP) Pesquisas em Geociências

PKP - Public Knowledge Project's Metadata Archive

Red liberoamericana de Revistas de Comunicacion y Cultura Em questão

Ulrich.s International Periodicals Directory

Psicologia: Reflexão e Crítica

Univerciência

Em questão

Zeitschriftendatenbank (ZDB)

Contingentia

\begin{tabular}{ll}
\hline & Fontes não localizadas \\
\hline Political Science and Government & Episteme \\
Social and Human Science Online Periodicals & Episteme, Psicologia: Reflexão e Crítica \\
Social Science Online Periodicals & Psicologia: Reflexão e Crítica \\
\hline
\end{tabular}

Fonte: Elaborado pelas autoras (2010).

característica geral das revistas interfere na sua visibilidade, além de limitar - mas não impossibilitar - o ingresso em fontes de informação de âmbito internacional.

\section{Mapeamento e análise das fontes de informação}

No Quadro 2, estão listadas as revistas e as respectivas fontes em que estão presentes, seguindo a classificação proposta por Rowley (2002).

Ao total foram relacionadas em um primeiro momento 75 fontes de informações utilizadas pelos periódicos do PPCU, além do Portal de Periódicos da Capes. Porém, ao organizar os dados, chegou-se ao número de 61 diferentes fontes, tendo em vista que algumas informações estavam duplicadas na descrição da revista ou a mesma fonte aparecia com a nomenclatura diferente. Pela descrição dessas fontes, foi possível constatar que 46 são bases de dados referenciais (35 bibliográficas e 11 catalográficas), uma base de dados de fontes e 11 portais, diretórios de revistas e outros tipos de fontes de informação.

Das 61 fontes, 20 estão arroladas no PPC e 41 não foram localizadas. Para descrever as fontes que não foram localizadas no PPC foi realizada pesquisa no mecanismo de busca Google. Porém, o resultado do PPC possui maior número de informações, pois, além de possibilitar acesso à fonte, fornece o tipo e possibilita acesso àqueles que solicitam assinatura. O insucesso na pesquisa, tanto no PPC como no Google, ocorreu com três fontes, que pode ser considerado um número baixo no universo dessa pesquisa, as quais não foram classificadas nem descritas na análise.

A Figura 1 ilustra, pelo gráfico de nuvens, as fontes em que estão presentes as revistas do PPCU, enfatizando aquelas que têm maior frequência de participação.

As fontes com menor ocorrência, presentes uma vez entre todas as revistas do PPCU, estão em um número considerável, já que diversas delas são fontes de informação específicas de uma determinada área do conhecimento. Os tipos variados de fontes enriquecem a presença dos periódicos do PPCU na Web. Identificaram-se diretórios, portais, catálogos de bibliotecas, entre outras fontes, nacionais e internacionais. Porém, as bases de dados bibliográficas, em especial as que possibilitam análises de produção científica através dos índices de citação, são pouco expressivas, como pode ser observado na Figura 1.

Há predominância de fontes multidisciplinares e com abrangência regional, neste caso, específico à América Latina. Sendo a indexação em índices referenciais internacionais um dos atributos que promovem a visibilidade, parte dos periódicos da Universidade necessita se adequar aos critérios de qualidade que são exigidos 


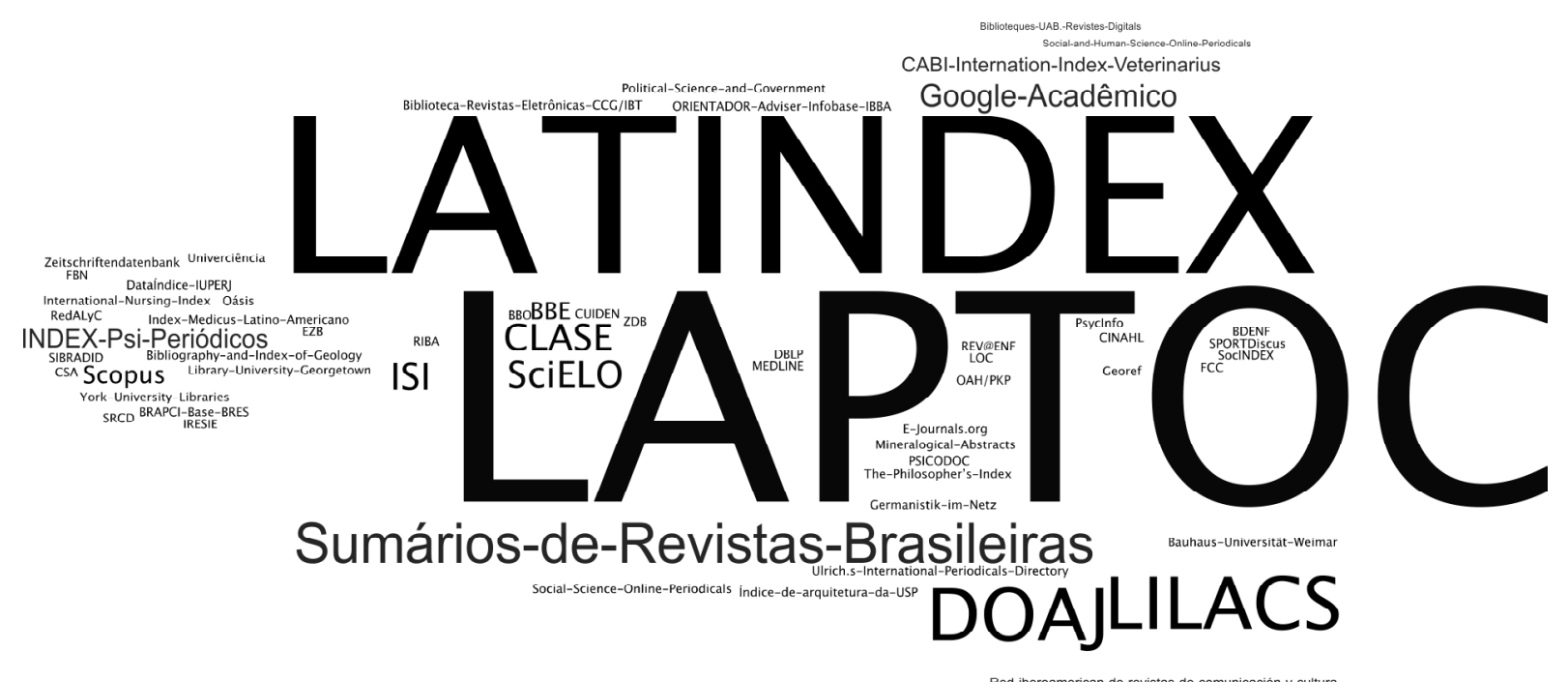

Figura 1. Nuvem: fontes de informação das revistas do PPCU.

Fonte: Elaborado pelas autoras (2011).

por essas bases se desejarem atingir uma audiência internacional. A adequação também possibilitará a conferência de melhorias do periódico de uma forma geral.

\section{A Latin American Periodicals Tables of Contents} (LAPTOC) é a fonte de informação na qual se encontra o maior número de revistas - 24 títulos - e a Latindex vem logo em seguida, tendo 18 títulos presentes na base. Tal ocorrência é explicável pela análise dos documentos de área da Capes: a área de Ciências Sociais Aplicadas I, por exemplo, sugere a presença nessas fontes de informação. Outras fontes que receberam destaque foram o Directory of Open Access Journals (DOAJ) (7 títulos) e o Lilacs (5 títulos).

Quanto à presença em portais de informação e diretórios de revistas, têm destaque as fontes Latindexe DOAJ. Ambas não indexam artigos de periódicos, mas listam e direcionam ao site das revistas e possuem acesso aberto. No DOAJ, a presença de revistas que utilizam o SEER/OJS é marcante. Na plataforma, há um plug-in de exportação de dados para o DOAJ, o que pode estimular os editores a submeterem proposta para participarem desta fonte. Já que a maioria das revistas utiliza o SEER/OJS para editar e gerenciar suas edições, esperava-se um número maior, porém entre os motivos para a ausência pode ser justamente o desinteresse de determinadas áreas em estar presente em portais, concentrando esforços para a indexação em bases de dados.
É importante ressaltar que as fontes de informação que não pertencem a tipologia das bases de dados bibliográficas não alcançam a mesma importância perante a comunidade científica, que é estimulada a ler e publicar periódicos indexados em bases de dados internacionais, sob responsabilidade de determinadas editoras, entre outras características qualificadoras. O idioma da fonte, então, é importante e, uma vez que a abrangência é internacional, será predominante o inglês. As fontes regionais podem também apresentar os dados no idioma inglês, mas observa-se em algumas delas a predominância do idioma espanhol. As fontes sob responsabilidade da Universidade Nacional Autónoma do México (UNAM) ilustram essa situação. Em contraponto, a Laptoc usa em sua base o idioma inglês, embora a maioria dos países participantes utilize o idioma espanhol.

O Google Acadêmico ainda é pouco valorizado pelas revistas do PPCU como fonte de informação, porém o potencial da ferramenta merece atenção. Já apontado por Jacso (2005) e Thelwall (2008) como uma ferramenta que oferece serviços similares às bases como o ISI e o Scopus, o uso do Google Acadêmico possibilita estudos bibliométricos que não poderiam ser executados em determinados periódicos do PPCU e de grande parcela das revistas brasileiras. 
Uma fonte de informação abundantemente consultada para a caracterização dos periódicos foi o catálogo de bibliotecas da UFRGS, o SABI. Além dos dados encontrados no CCN, foi possível recuperar diversas informações relacionadas às fontes de informação em que estão presentes as revistas da Universidade e ainda artigos de determinadas publicações, como a Educação \& Realidade. Nenhum periódico aponta a presença no $\mathrm{SABI}$, entretanto apontam para catálogos de outras bibliotecas internacionais ou de instituições importantes no Brasil. Possivelmente, estar presente em um catálogo de uma importante biblioteca é considerado como prestígio para a revista pelos editores e pelos usuários, porém foi verificado que apenas o título da publicação está catalogado, não possibilitando recuperação do conteúdo dos artigos.

Um ponto que foi observado durante a análise das fontes diz respeito à adaptação dos serviços de determinadas bases de dados, fato ocorrido graças às TIC. Se antes as bases de dados bibliográficos não forneciam acesso ao texto completo, agora, elas fornecem o link para o usuário acessá-los integralmente. No SABI também é possível recuperar o artigo quando ele está online e, sendo uma produção intelectual da UFRGS, o LUME (Repositório Institucional da Universidade) possibilita também acesso ao texto. Por esse motivo, a inserção de novas características aos serviços já existentes pode ser explicada pela dificuldade para identificar a diferença entre as fontes de informação.

Além do aumento do número de revistas nas últimas décadas, pode ser observado o surgimento de novas fontes para poder compilar a informação contida naquelas. A quantidade de portais, diretórios de revistas e bases de dados que surgiram em meados dos anos 2000 foi observada na descrição das fontes. Inclusive, pode-se dizer que há duplicidade naquelas criadas com a metodologia da SciELO. Esse pode ter sido um recurso da área do conhecimento para estar presente em mais fontes de informação, já que a quantidade também parece ser estimulada pelas diversas áreas. Contudo, volta-se à questão sobre a qualidade das fontes: nem sempre a quantidade é tão importante quanto estar em uma base valorizada pela comunidade científica.

Job et al. (2008) sugerem que as revistas da área de Educação Física sejam mais competitivas em relação às publicações das Ciências da Saúde ao qualificarem suas publicações. Tal sugestão é válida para as revistas do PPCU: buscarem qualificação em relação às revistas da mesma grande área, procurando alcançar os níveis de qualidade daquelas consideradas as mais importantes dentro de sua área, preferencialmente, das publicações nacionais ou não - com abrangência internacional, que possuam visibilidade na ciência e que sejam bem avaliadas. Ao se qualificarem a exemplo das revistas de prestígio, poderão ser bem avaliadas - relembrando que as áreas do conhecimento possuem documentos diferentes na Capes e os níveis de exigência para receber um melhor estrato Qualis são distintos. Já as fontes de informação possuem critérios diversos de avaliação para inserção do periódico em sua base, tendo em vista a diversidade de tipos, de abrangência, de cobertura, de idiomas, entre outras características que elas possuam.

Os sistemas de avaliação, sejam os de produção científica, sejam os critérios das fontes de informação ou de qualquer natureza relacionada ao meio acadêmico, são constantemente criticados, discutidos e melhorados de acordo com a realidade e as mudanças da ciência. Tendo como base essa ideia, é interessante que a atribuição de visibilidade para um periódico não seja limitada à presença em determinados índices e bases de dados, uma vez que as áreas do conhecimento são diferentes entre si. Um instrumento adequado às ciências duras nem sempre pode ser considerado para as Ciências Humanas. A presença em fontes diversificadas na Internet possibilita um grau de visibilidade a essas publicações pode-se incluir, também, o acesso aberto, a capacidade de recuperação da informação ou ainda a buscabilidade na Web.

\section{A visibilidade das revistas}

A análise desse item foi feita após a classificação das revistas em grandes áreas, tendo em vista que uma análise individual não demonstraria tendências, mas sim a situação de cada título de revista. A análise por grandes áreas do conhecimento possibilitou a visão geral do grupo de revistas e evidenciou a concentração de publicações na mesma área do conhecimento. O fato de haver muitas publicações em uma mesma temática pode interferir na visibilidade geral da área, pois, assim como 
há periódicos bem classificados, presentes em diversas fontes de informação de diferentes tipos e qualidades, entre outros atributos positivos, há revistas que não possuem características tão positivas. Geralmente são periódicos recém lançados ou que possuem alguma particularidade em relação à periodicidade.

Entende-se também que, dentro de uma mesma área do conhecimento, há temáticas específicas, podendo ser esta a explicação, em alguns casos, para a existência de mais de um periódico dentro de uma faculdade, instituto ou programa de pós-graduação. Essa situação pode ser confirmada ao verificar que, se dentro de uma mesma unidade há duas ou mais publicações, os setores responsáveis são diferentes. Pode ainda existir uma demanda de submissão de artigos em que seja necessário mais de um título para possibilitar a sua publicação. Ainda assim, é questionável a existência de duas revistas, da mesma área do conhecimento, destinada ao mesmo público-alvo e disciplinas, editadas na mesma Faculdade e com características semelhantes.

A área de Ciências Humanas se destaca pelo número de revistas existentes e por elas possuírem atributos almejados pelos editores, como a presença em fontes de informação e estratificação alta. Já na área de Ciências da Saúde foi constatada uma grande discrepância entre as revistas, constando aquelas com excelentes condições de visibilidade e outras que precisam melhorar expressivamente para se tornarem mais visíveis pelos usuários da área. Nas Ciências Exatas e da Terra, o resultado da análise apontou que as revistas alcançam alguma visibilidade, porém há pontos frágeis no atendimento dos atributos que deixam o grupo menos valorizado. Um desses pontos é a existência de uma revista nova e não classificada ainda no Qualis. Além disso, possui poucos periódicos para uma área que valoriza as publicações de eventos e o Journal Citation Reports (JCR) ao mesmo tempo.

As Ciências Sociais Aplicadas também não demonstraram uniformidade, porém as subáreas existentes são particularmente diferentes entre si. Já a análise de revistas interdisciplinares é um desafio, tendo em vista a grande diferença entre as publicações. Embora recebam artigos de diversas áreas, os títulos possuem características bastante diferenciadas entre si e atingem públicos específicos para cada revista ou mesmo por edição, no caso desta ser temática.

Quanto à disponibilização de conteúdos na Internet, o acesso aberto é soberano entre as publicações e a presença em diferentes fontes de informação na Web contribui positivamente. Gruszynski et al. (2007) verificaram que a prioridade de um grupo de periódicos, na primeira metade dos anos 2000, era obter visibilidade pela presença na Internet. Finalizada essa etapa - afirmativa possível ao retomar que todas as revistas da Universidade são de acesso aberto - ainda há a necessidade da presença maior em índices bibliográficos. Villamón et al. (2009) identificaram revistas que disponibilizam fascículos somente no formato impresso, porém estão presentes em índices, disponibilizando a referência e, algumas vezes, o resumo. Assim, verifica-se que a presença na Web é importante para estar presente nessas fontes de informação, mas não é fundamental.

Sobre o idioma de publicação, verifica-se que as revistas aceitam artigos em português, inglês e espanhol, na sua maioria, tendo títulos com artigos em francês e alemão. O SEER/OJS permite que a publicação seja traduzida para diversos idiomas, o que beneficia a equipe no momento que deseja disponibilizar o site da revista em outras línguas. Porém, se mapeado cada artigo das revistas, é possível observar a presença quase exclusiva de artigos em português, com resumo em inglês ou espanhol, e alguns artigos em outras línguas, geralmente de autores convidados. Embora a situação só pudesse ser confirmada com um estudo mais aprofundado e com a demonstração de dados, a primeira análise das revistas, no momento da caracterização, permitiu inferir a situação descrita acima. $O$ inglês é o idioma reconhecido pelas comunidades internacionais, mas a publicação em língua portuguesa torna a revista mais atraente para determinados públicos, que leem e compreendem melhor o idioma local. Assim, a revista possui visibilidade nos locais onde a língua é falada.

Analisando a presença em índices bibliográficos, foi possível observar que a grande maioria das revistas está presente em mais de uma dessas fontes de informação, de tipos variados. Porém, se a quantidade pode ser uma característica positiva para as revistas, a qualidade, verificada na abrangência geográfica, idioma e função da fonte de informação (se indexa artigos ou se apenas aponta para os títulos), são indispensáveis para 
que ocorra a visibilidade. A ocorrência em um maior número de fontes de qualidade pode auxiliar em outras características positivas das revistas, como a avaliação do Qualis e a aquisição de credibilidade entre a comunidade científica e comitês avaliadores de outras bases de dados. As duas fontes mais utilizadas pelas revisas do PPCU são multidisciplinares e com abrangência geográfica na América Latina, quais sejam, a Latindex e a LAPTOC. Em comum, ambas oferecem acesso aberto ao seu conteúdo, não exigindo assinatura ou cadastro. Assim, sobre a qualidade das bases de dados em que estão presentes os periódicos da Universidade, pode-se constatar que, embora consistente naquelas de abrangência regional, ainda é escassa a presença nos índices de abrangência internacional, embora tenha ocorrido o crescimento de títulos brasileiros no ISI, por exemplo. Também não foi verificada a presença marcante em bases de dados específicas das áreas do conhecimento, sendo as multidisciplinares a grande maioria.

\section{Conclusão}

De um modo geral, as revistas da UFRGS apresentaram características promotoras de visibilidade. Se a análise da visibilidade fosse restrita à presença nas bases de dados internacionais, poucos títulos poderiam ser considerados visíveis à comunidade científica. Sob o ponto de vista de que a visibilidade é composta por uma série de características ou atributos, especificamente pela publicação eletrônica na Internet, acesso aberto, buscabilidade, idioma, presença nas fontes de informação e avaliação, certamente em diversos aspectos são necessárias melhorias. Especialmente no que concerne à presença em índices referenciais de âmbito internacional, o que envolveria mudanças expressivas nas publicações, em especial quanto ao idioma. Porém em relação a outras características, como o acesso aberto e a capacidade de recuperar a informação em mecanismos de buscas e tipos variados de fontes de informação, as revistas da Universidade estão bem colocadas.

Na literatura sobre visibilidade da produção científica, destaca-se a visão que a resume à presença na Web, ou capacidade em ser recuperada pelos mecanismos de busca. Alguns estudos mencionam a visibilidade dos periódicos, mas não definem um conceito para apontar o que é ser visível, enquanto outros utilizam métodos que consideram poucas características para definir visibilidade. Todavia, estudos sobre a avaliação e caracterização de periódicos estão cada vez mais presentes na literatura, sob as óticas das diversas áreas do conhecimento, mas em especial à da Ciência da Informação.

Durante o levantamento bibliográfico sobre a visibilidade de periódicos, percebeu-se que os artigos relevantes são principalmente escritos em idiomas de países periféricos, quais sejam, português e espanhol. Infere-se, com isso, que nesses países há uma preocupação maior com a visibilidade das publicações periódicas. Isso se deve, provavelmente, ao fato que a maioria de suas publicações não consta em bases de dados bibliográficas de alcance internacionais, como Web of Science. Ainda assim, as publicações brasileiras estão conquistando um espaço maior nessas fontes de informação, mesmo que a presença dos periódicos nacionais seja pequena se comparada aos países com tradição em publicar no idioma inglês.

Percebe-se grande valorização da comunidade científica em relação aos periódicos que estão presentes em bases de dados internacionais. Certamente uma revista que está presente nas importantes fontes de informação possui qualidade. Por outro lado, observou-se que há diversas críticas aos produtos gerados por essas bases, em especial aos indicadores de produção, além das coberturas geográficas e de assunto. Se o ISI tem na sua maioria revistas norte-americanas e inglesas da área das ciências duras, é a fonte mais adequada às revistas brasileiras das demais áreas? O público-alvo dessa área a utilizaria para consultar artigos de seu interesse?

Essas indagações não procuram minimizar a importância dos periódicos presentes no ISI, Scopus ou quaisquer importantes fontes de informação. Ao contrário, enfatiza-se a importância da visibilidade internacional. Porém, o ponto que se deseja abordar é que embora não tenha atingido esse patamar, a ciência publicada nas revistas científicas da UFRGS não é invisível: está na Web, com acesso aberto, pode ser recuperada nos mecanismos de busca e está presente em diversas fontes de informação.

Constata-se que o tema visibilidade é muito utilizado, mas ainda pouco explorado na literatura científica. Considera-se que este estudo pode gerar outras formas de abordar a mesma temática ou ser
189

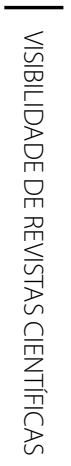


corroborado por trabalhos similares. Foi possível concluir que a visibilidade é formada por uma série de atributos, sendo que os mais relevantes para esta análise foram: a publicação eletrônica na Internet e com acesso aberto, o idioma de publicação da revista, as bases de dados referenciais nacionais e internacionais, a buscabilidade e a presença em outras fontes de informação. Essas, pode-se afirmar, estão, em grande parte, presentes nas revistas do Portal de Periódicos Científicos da UFRGS.

Para o aumento da visibilidade das revistas da Universidade é necessário o trabalho em equipe. Assim, recomenda-se: às equipes editoriais buscarem melhorias dos aspectos técnicos das revistas; atrair a submissão de artigos de qualidade e atender aos rigorosos critérios das fontes de informação; aos autores, o envio de artigos de qualidade; às unidades de informação, participar não só na formatação do artigo, mas indicando as fontes de informação adequadas e, quando possível, trabalhando junto à comissão editorial. Enfim, o conjunto desses fatores, entre outros, aumentará a visibilidade dos periódicos presentes no Portal de Periódicos Científicos da UFRGS e, por extensão, nos demais portais de revistas científicas.

\section{Referências}

Aguado-López, E.; Rogel Salazar, R.; Becerril-Garcia, A. Limitese potencialidades da avaliação científica: crítica epistemológica à cobertura de bases de dados è construção de indicadores. In: Ferreira, S.M.S.P.; Targino, M.G. (Org.). Acessibilidade evisibilidade de revistas científicas eletrônicas. São Paulo: Senac, 2010. p.175-212.

Céndon, B.V. Serviços de indexação e resumo. In: Campello, B.S. et al. (Org.). Fontes de informação para pesquisadores e profissionais. Belo Horizonte: Editora UFMG, 2000. p.217-248.

Gruszynski, A.C.; Golin, C.; Lucchese, A. Desafios para a comunicação da ciência: um estudo sobre os periódicos científicos e eletrônicos da UFRGS. In: Congresso Brasileiro de Ciência da Comunicação, 30., 2007, Santos. Anais eletrônicos... Santos: Intercom, 2007. p.1-15. Disponível em: <http://www.intercom. org.br/papers/nacionais/2007/resumos/R1395-1.pdf>. Acesso em: 12 maio 2012.

Hernández Ortega, B. et al. Efecto de la calidad de un sitio web sobre el tráfico recibido: el caso de la banca electrónica en España. Information Research, v.15, n.2, 2010. Disponible en: <http://informationr.net/ir/15-2/paper429.html>. Acceso en: 22 set. 2010.

Jacso, P. As we may search: Comparison of major features of the Web of Science, Scopus and Google Scholar citationbased and citation-enhanced databases. Current Science, v. 89, n.9, p.1537-1547, 2005. Available from: <www.ias.ac.in/ currsci/nov102005/1537.pdf>. Cited: Nov. 10, 2010.

Job, I.; Fraga, A.B.; Molina Neto, V. Invisibilidade das revistas brasileiras de educação física nas bases de dados. Cadernos de Biblioteconomia, Arquivística e Documentação, v.1, p.14-26, 2008.

Meneghini, R. Avaliação da produção científica e o projeto ScielO. Ciência da Informação, v.27, n.2, p.219-20, 1998. Disponível em: <http://www.scielo.br/pdf/ci/v27n2/meneghini. pdf>. Acesso em: 24 abr. 2010.

Miguel, S.; Herrero-Solana, V. Visibilidad de las revistas latinoamericanas de bibliotecología y ciencia de la informacióna través de Google Scholar. Ciência da Informação,

v.39, n.2, p.54-67, 2010. Disponible en: <http://revista.ibict.br/ index.php/ciinf/article/view/1722>. Acceso en: 28 fev. 2011.

Packer, A.L.; Meneghini, R. Visibilidade da produção científica. In: Poblacion, D.A.; Witter, G.P.; Silva, J.F.M. (Org.). Comunicação \& produção científica: contexto, indicadores e avaliação. São Paulo: Angellara, 2006. p.237-259.

Peazê, L. Google vs. Jornais = contextualização. Observatório da Imprensa, ano15, n.56, 2009. Disponivel em: <http://www. observatoriodaimprensa.com.br/artigos.asp?cod=567 JDB014>. Acesso em: 11 out. 2010.

Rowley, J.E. A biblioteca eletrônica. Brasília: Briquet de Lemos, 2002.

Santos, S. Sistema SciELO de publicação eletrônica. In: Encontro Nacional de Editores Científicos, 10., 2005, São Pedro, SP. Anais eletrônicos... 2005. Disponível em: <http://www. eventos. bvsalud.org/abec/public/documents/Solange_Sistema SciELO_OJS-094024.pdf>. Acesso em: 30 mar. 2012.

Silva, J.F.M.; Ramos, L.M.S.V.C.; Noronha, D.P. Base de dados. In: Poblacion, D.A.; Witter, G.P.; Silva, J.F.M. (Org.). Comunicação \& produção científica: contexto, indicadores e avaliação. São Paulo: Angellara, 2006. p.261-286.

Thelwall, M. Bibliometrics to webometrics. Journal of Information Science, v.34, n.4, p.605-621, 2008. Available from: <http://jis.sagepub.com/cgi/content/abstract/34/4/605>. Cited: Nov. 30, 2010.

Villamón, M. et al. Las revistas españolas de ciencias del deporte incluidas en el catálogo Latindex. Movimento, v.15, n.3, p.13-34, 2009. Disponible en: <http://seer.ufrgs.br/ Movimento/article/download/8567/5577>. Acceso en: 11 nov. 2010.

Zimba, H.F.; Mueller, S.P.M. Colaboração internacional e visibilidade científica de países em desenvolvimento: o caso da pesquisa na área de medicina veterinária em Moçambique. Informação e Sociedade, v.14, n.1, p.45-68, 2004. Disponível em: <http://www.ies.ufpb.br/ojs2/index.php/ies/article/view/ 71/1544>. Acesso em: 12 jun. 2010. 
